\title{
Riccardo Benedettini, Felice Gambin (a cura di), Carlo Bo e la letteratura del Novecento. Da Valéry a García Lorca
}

\section{Francesca Forcolin}

\section{(2) OpenEdition \\ Journals}

\section{Edizione digitale}

URL: http://journals.openedition.org/studifrancesi/4523

DOI: $10.4000 /$ studifrancesi.4523

ISSN: 2421-5856

\section{Editore}

Rosenberg \& Sellier

\section{Edizione cartacea}

Data di pubblicazione: 1 settembre 2016

Paginazione: 387-388

ISSN: 0039-2944

\section{Notizia bibliografica digitale}

Francesca Forcolin, « Riccardo Benedettini, Felice Gambin (a cura di), Carlo Bo e la letteratura del Novecento. Da Valéry a García Lorca », Studi Francesi [Online], 179 (LX | II) | 2016, online dal 01 septembre 2016, consultato il 18 septembre 2020. URL : http://journals.openedition.org/studifrancesi/ 4523 ; DOI : https://doi.org/10.4000/studifrancesi.4523

Questo documento è stato generato automaticamente il 18 settembre 2020.

\section{cc) $(8)$}

Studi Francesi è distribuita con Licenza Creative Commons Attribuzione - Non commerciale - Non opere derivate 4.0 Internazionale. 


\title{
Riccardo Benedettini, Felice Gambin (a cura di), Carlo Bo e la letteratura del Novecento. Da Valéry a García Lorca
}

\author{
Francesca Forcolin
}

\section{NOTIZIA}

RICCARDO BENEDETTINI, FELICE GAMBIN (a cura di), Carlo Bo e la letteratura del Novecento. Da Valéry a García Lorca, Edizioni dell'Orso, Alessandria, 2015, 140 pp.

1 A quindici anni dalla scomparsa di Carlo Bo (l'anniversario ricorre nel 2016), il suo apporto critico preserva intero il suo spessore: per l'attualità, per l'impatto sugli studi contemporanei, ma soprattutto per l'attività di divulgatore di opere e scrittori ancora sconosciuti nell'Italia dell'epoca fascista. Un lavoro pioneristico, quello di Bo, che ripercorre efficacemente il volume diretto da Riccardo BENEDETTINI e Felice GAMBIN, frutto di un convegno in sua memoria tenutosi nel 2011 a Verona. Dello studioso ligure, nei sette contributi che compongono la miscellanea, trapela un'estrema lucidità, un'attività addirittura rivoluzionaria che si destreggia tra critica letteraria e traduzione (fu il fondatore della "Scuola per Interpreti e traduttori" di Milano nel '51), tra Italia, Francia e Spagna.

2 «Carlo Bo francesista e ispanista», è l'icastico incipit di Benedettini e Gambin nell'introduzione che porta lo stesso titolo (pp. 1-11), condensando l'obiettivo e la struttura dell'intero volume. Ci troviamo di fronte infatti a due parti nettamente distinte: la prima comprende i primi quattro articoli e riguarda la metà francese di Bo; i successivi tre quella spagnola, facendoci scoprire un critico e traduttore dalla doppia e vasta specializzazione.

3 Sara ARENA, con "Arrivare alla riva dell'eternità". Spiritualità e letteratura nella meditazione di Carlo Bo su Paul Claudel (pp. 13-27), apre il dibattito concentrandosi sul "caso Claudel", sulla lettura cioè che fa Carlo Bo di questi scritti della meditazione e della spiritualità. 
Se in numerose recensioni degli anni Trenta l'opera di Claudel viene criticata per lo stile ridondante, la struttura debole, l'incrocio di immagini liturgiche e carnali, la posizione di Bo appare controcorrente: egli intende questa opera, infatti, come un corpus unico da comprendere attraverso un vero e proprio "ascolto" da parte del lettore, che vi si deve avvicinare, tendere l'orecchio, porsi a fianco dell'autore per capire appieno le sensazioni e lo stato d'animo claudeliano. Per fare ciò, Bo si discosta dai classici strumenti della critica letteraria leggendo l'autore da credente e guardandolo da una certa distanza nel suo intimo colloquio con Dio, colloquio evidente nel testo autobiografico $\mathrm{Ma}$ conversion, il più amato da Bo, simbolo di una totale adesione all'eterno.

4 Tania Collani, in Carlo Bo lettore dei surrealisti francesi: la poesia, la vita e la verità (pp. 29-46), sposta l'attenzione sul Surrealismo, nei cui confronti Bo ebbe un atteggiamento rivoluzionario: anche in questo caso fu una voce fuori dal coro rispetto ai suoi contemporanei perché, allontanandosi dalle tendenze critiche degli anni Trenta, promosse e difese le sperimentazioni del gruppo, sottolineando le grandi qualità da prosatore di Breton. Ai suoi occhi erano opere fresche e spontanee, dalla carica vitale e spirituale. Nel contributo proposto, Collani delinea gli ostacoli che Bo dovette superare in questo suo percorso, dettati innanzitutto dall'ostilità nei confronti delle innovazioni francesi e in generale delle importazioni culturali durante l'epoca fascista.

5 Passiamo poi a Laura colombo che in "Orfeo nero" visto da Carlo Bo: l'“Antologia di poeti negri" (pp. 47-66) si concentra sulla letteratura del "mondo nero" largamente riconosciuta dall'inizio del Novecento e in particolare dal '48, il centenario dall'abolizione della schiavitù in Francia - siglata dalla pubblicazione dell'Anthologie di Senghor, uno dei maggiori promotori della négritude, con prefazione firmata da Sartre. Stimolato da questa "letteratura-mondo", Bo ne promuove la diffusione in Italia attraverso la sua Antologia, in cui presenta, grazie a un itinerario geografico e cenni biografici degli autori, una serie di nomi poco noti nella nostra penisola, originari dell'America e dell'Africa: ma l'interesse del volume risiede soprattutto sui problemi e sulle difficoltà che inevitabilmente compaiono quando si traducono poemi impregnati di una cultura "altra", ricchi di sostrati a noi lontani tra cui dialetti, ritmi e suoni.

Chiude la sezione francese il contributo di Fabio scotTo, L'idea del Novecento poetico francese in Carlo Bo: Valéry, Supervielle, Michaux (pp. 67-86), contributo di più ampio respiro dove si esamina il Carlo Bo lettore della poetica francese d'inizio Novecento. Come afferma Scotto, per Bo il lettore è una figura centrale, è il «vero autore di una forma di lettura che è condizione stessa dell'esistenza e della ricezione dell'opera». Se Bo individua tra le voci più autorevoli del secolo Rivière, Gide, Apollinaire e Sartre, un approccio più controverso lo ebbe nei confronti di Valéry, letto mediante Pascal, di cui Bo diversamente da Valéry è un grande estimatore: l'attenzione va soprattutto alla produzione in prosa dell'autore, prima tra tutte Monsieur Teste comparato al Des Esseintes di Huysmans. L'approccio fu difficile anche con Supervielle, un autore che elude i problemi lasciandosi andare a una «leggerezza giocosa». Si passa poi al Bo lettore di Michaux, poeta dalla scrittura fresca, profonda e ancestrale, autonomo rispetto al surrealismo, e dalla «natura eccezionale».

7 Apre la sezione spagnola il contributo molto sentito di Maria Grazia PROFETI, Il Lorca di Carlo Bo (pp. 87-100). Gli anni della guerra civile spagnola portarono a una maggiore consapevolezza, in Italia, di quella realtà, facendone riscoprire la cultura e più in particolare la poesia di Lorca soprattutto quando si apprese la notizia della fucilazione. 
Bo fu tra i divulgatori della sua opera, grazie a un'introduzione alle poesie e alla traduzione dei versi del poeta, nonostante la versione italiana talvolta manchi di ritmo, abbia sviste e imprecisioni come dimostra Maria Grazia Profeti che ci propone una propria interpretazione.

Dalla traduzione si passa alla produzione critica di Bo grazie a Paola BELLOMI, in La ricezione della letteratura spagnola in Italia: il contributo di Carlo Bo (pp. 101-114). Bo ebbe un primo contatto con la produzione spagnola negli anni Trenta recensendo Gracián; rimase affascinato dalla Generazione del '27 e dai loro maestri, come Jiménez o Machado, con cui il rapporto fu però più conflittuale. Le critiche di Bo sono tuttora un punto di riferimento, nonostante siano avulse dal contesto storico delle opere, un'astoricità che nel tempo non ha attecchito; e nonostante il suo punto di vista negli anni sia cambiato per la maturità anagrafica e intellettuale, soprattutto riguardo a Machado.

9 Felice GAMBIN focalizza l'attenzione in maniera convincente e precisa sul rapporto tra Bo e la prosa spagnola, prendendo in esame in particolare tre autori in Le fatiche dell'infaticabile ispanista Carlo Bo: Ganivet, Unamuno, Ortega y Gasset (pp. 115-134). Bo fu traduttore e critico di Ganivet, sollecitato dal comune interesse per il tema della ricerca della verità sull'uomo e della spiritualità; dedica ampio spazio a Unamuno in un rapporto che mescola ammirazione e critica, facendo conoscere le sue opere al pubblico italiano grazie alla traduzione di un'antologia poetica; ma uno dei più grandi meriti di Bo fu quello di aver introdotto attraverso una delle prime traduzioni italiane la scrittura di Ortega y Gasset.

10 A chiudere ogni articolo troviamo una bibliografia, e in fondo al volume l'indice dei nomi. 